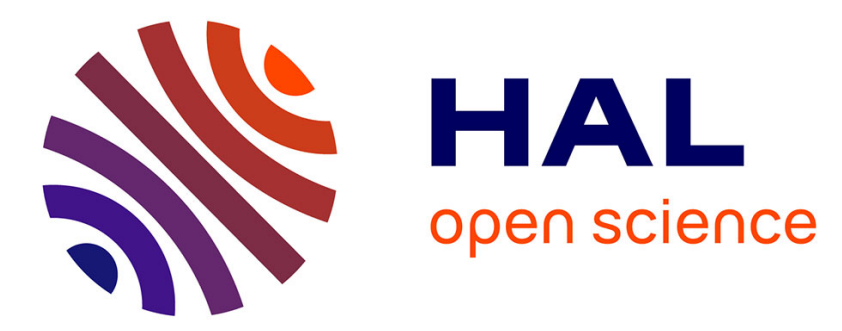

\title{
Custom surgical management of invasive malignant tumors of the scalp
}

Pauline Marijon, Chloé Bertolus, Jean-Philippe Foy, Guillaume Marechal, Jean-Baptiste Caruhel, Mourad Benassarou, Alexandre Carpentier, Vincent Degos, Aymeric Amelot, Bertrand Mathon

\section{To cite this version:}

Pauline Marijon, Chloé Bertolus, Jean-Philippe Foy, Guillaume Marechal, Jean-Baptiste Caruhel, et al. Custom surgical management of invasive malignant tumors of the scalp. Acta Neurochirurgica, 2020, 10.1007/s00701-020-04525-0 . hal-02946424

\section{HAL Id: hal-02946424 https://hal.sorbonne-universite.fr/hal-02946424}

Submitted on 23 Sep 2020

HAL is a multi-disciplinary open access archive for the deposit and dissemination of scientific research documents, whether they are published or not. The documents may come from teaching and research institutions in France or abroad, or from public or private research centers.
L'archive ouverte pluridisciplinaire HAL, est destinée au dépôt et à la diffusion de documents scientifiques de niveau recherche, publiés ou non, émanant des établissements d'enseignement et de recherche français ou étrangers, des laboratoires publics ou privés. 
3 Pauline MARIJON ${ }^{1}$, Chloé BERTOLUS ${ }^{2,3}$, MD, PhD; Jean-Philippe FOY ${ }^{2,3}$, MD; Guillaume

4 MARECHAL ${ }^{2}$, MD; Jean-Baptiste CARUHEL ${ }^{2}$, MD; Mourad BENASSAROU ${ }^{2}$, MD;

5 Alexandre CARPENTIER ${ }^{1,3,4}$, MD, PhD; Vincent DEGOS ${ }^{3,5}$, MD, PhD; Aymeric AMELOT ${ }^{1,3}$,

6 MD, PhD; Bertrand MATHON ${ }^{1,3,4}$, MD.

7

81 AP-HP, La Pitié-Salpêtrière - Charles Foix University Hospital, Department of Neurosurgery, F-

9 75013, Paris, France

102 AP-HP, La Pitié-Salpêtrière - Charles Foix University Hospital, Department of Maxillo-Facial

11 Surgery and Stomatology, F-75013, Paris, France

123 Sorbonne University, UPMC Univ. Paris 06, F-75005, Paris, France

134 Brain and Spine Institute (ICM; INSERM, UMRS 1127; CNRS, UMR 7225), Paris, France

145 AP-HP, La Pitié-Salpêtrière - Charles Foix University Hospital, Department of Anesthesia and

15 Critical Care, F-75013, Paris, France

16

Short running head: Surgery of invasive cancers of the scalp

18

19

Corresponding author:

Dr Bertrand MATHON, MD, MSc.

21 Department of Neurosurgery, Groupe Hospitalier Universitaire de la Pitié-Salpêtrière

22 47-83, Boulevard de l'Hôpital 75013 Paris, France.

23 Email: bertrand.mathon@aphp.fr

ORCID: 0000-0002-9182-5846

25

26 
ABSTRACT

Background: There is no universal management protocol concerning invasive malignant tumors of the scalp with bone and dura mater invasion. The aim of this study was to report and discuss our experience in the management of these forms of tumors.

Methods: We retrospectively reviewed all consecutive patients of microsurgical scalp reconstruction performed after resection of invasive cutaneous malignancies of the scalp, calvarium and dura mater from 2017 to 2019, at Pitié-Salpêtrière University Hospital (Paris, France).

Results: Five patients met inclusion criteria. There were 3 squamous cell carcinomas and 2 undifferentiated sarcomas. Mean age at surgery was 63.6 years. The sex ratio male/female was 4 . Two received radiation prior to resection and 2 patients had a history of prior scalp tumor surgery. All the patients underwent craniectomy and the mean cranial defect size was $41 \mathrm{~cm}^{2}$. Cranioplasty was performed in one patient. Soft tissue coverage was provided by free tissue transfer of latissimus dorsi muscle in all patients. In 4 patients, split-thickness skin graft was performed in a second surgical stage few weeks later. There were no intraoperative complications and no complications into the donor site for the tissue transfer or the skin graft. Two patients had flap necrosis that healed after a new free flap of latissimus dorsi.

Conclusions: Wide resection with craniectomy, and reconstruction with microvascular free tissue transfer provides safe and reliable treatment of recalcitrant invasive scalp skin cancers. The surgical management of these complex patients is a challenge that must be conducted by trained, experienced and multidisciplinary teams.

\section{KEYWORDS}

craniofacial microsurgery,

cranioplasty,

cutaneous malignancy,

53 free tissue transfer,

54 microsurgical reconstruction, neurosurgery. 


\section{INTRODUCTION}

Few data are available in the literature concerning scalp invasive malignancies and their surgical care (excision and reconstruction after large and complex resection of calvarium). Tumor excision with scalp and bone invasion can be a surgical challenge due to the size and the invasiveness of the tumor. Likewise, calvaria reconstruction after excision can be also challenging and is generally performed using skin grafts or large flap depending on the defect size. However, there is no universal management protocol for these forms of tumors. When they are invasive, craniectomy and dura mater excision may be required to obtain full tumor excision, which exposes the patient to a risk of cerebrospinal fluid leak and infection. After these procedures, there is no consensus on the need of cranioplasty after microsurgical reconstruction of the skin.

Reconstruction scalp and skull defect resulting from tumor excision present most difficulties for the surgeon. Wide excision may include skin, periosteum, skull and dura mater, creating a complex wound. The use of radiation therapy and chemotherapy can compromise healing and scalp's vascularization. Flaps used for reconstruction should be durable to support adjuvant treatment. In 1972, free tissue transfer for reconstruction of large scalp defect was used for the first time, with an omentum flap to cover the defect [15]. Since then, other series have demonstrated the efficacy of this approach $[16,23]$. The aim of the present study was to report and discuss our experience in the management of invasive malignant tumors of the scalp with bone and dura mater invasion.

\section{PATIENTS AND METHODS}

\section{Patients recruitment}

We retrospectively reviewed all consecutive patients of microsurgical scalp reconstruction performed after resection of invasive cutaneous malignancies of the scalp, calvarium and dura mater from February 2017 to July 2019, at Pitié-Salpêtrière University Hospital (Paris, France).

Presurgical evaluation included: complete general and neurological examination, brain MRI and CT-scan, body scanner and in some cases body positron emission tomography (PET). These data 
were analyzed by a multidisciplinary team, including plastic surgeon, neurosurgeon, anesthetist, oncologist, radiotherapist, dermatologist and radiologist, to determine whether surgical treatment was indicated and whether a postoperative treatment would be required.

\section{Classification of comorbidities and outcomes}

Patient's data were collected through review of medical records. Current smoking was defined as smoking within 2 months of surgery. Hypertension and hyperlipidemia were defined by the use of antihypertensive and cholesterol-lowering drugs, respectively. Diabetes was defined by the use of oral hypoglycemic drugs or insulin. Immunosuppression was defined by use of immunosuppressive drugs or diagnosis known to cause an immunocompromised state. Patient's preoperative physical status was classified using the American Society of Anesthesiologists (ASA) score. Patients with a mild systemic disease were classified as ASA II. Patients with a systemic disease which is not incapacitating were classified as ASA III, and patients with an incapacitating systemic disease which is life-threatening were classified as ASA IV.

Measurement of skull defects were performed on postoperative CT-scans. Patients were carefully followed to ensure that no complications were missed. Cerebral spinal fluid (CSF) leak was defined by the presence of fluid collected from the operative site. Partial flap loss was defined as flap necrosis that resulted in subtotal loss of flap, while complete flap loss was defined as requiring total flap replacement.

\section{Standard Protocol Approvals, Registrations and Patient Consents}

The database is registered with the Commission Nationale de l'Informatique et des Libertés $\left(\mathrm{n}^{\circ}\right.$. 2214386). In accordance with the ethical standards of our hospital's institutional review board, the Committee for the Protection of Human Subjects, and French law, written informed consent was not needed for demographic, physiological and hospital-outcome data analyses because this observational study did not modify existing diagnostic or therapeutic strategies; however, patients were informed of their inclusion in the study. The manuscript was prepared in accordance with the Strengthening the Reporting of Observational studies in Epidemiology (STROBE) statement. 
112

113

114

115

116

117

118

120

122

123

124

125

126

127

128

129

130

131

132

133

134

135

136

137

138

139

\section{RESULTS}

\section{Patient characteristics}

We found 5 patients who underwent resection followed by reconstruction surgery for invasive malignant tumors of the scalp extending into the calvarium and the dura mater. Mean age at surgery was $63.6 \pm 9.2$ years (range 35-84). The sex ratio male/female was 4. Preoperative patient comorbidities are summarized in Table 1 . The mean follow-up period was 19 (range 7-36) months.

\section{Tumor characteristics and surgical management}

Of the 5 patients included in the study, 3 had stage IVA squamous cell carcinomas and 2 had sarcomas (one grade III undifferentiated sarcoma and one low-grade myofibroblastic sarcoma). The 3 patients with squamous cell carcinomas presented both clinical and histological poor prognostic factors according to the National Comprehensive Cancer Network (NCCN, USA) classification [18]. Two patients were treated by radiation therapy prior to resection (\#1 and \#2) and 2 patients had a history of prior scalp tumor surgery (\#1 and \#5). None of the patients had adjuvant lymph node excision.

Two patients had active scalp infections at the time of presentation: one had methicillinresistant Staphylococcus aureus (MRSA) plus Proteus mirabilis plus Enterococcus Faecalis extra- and subdural empyema with skull necrosis that required surgical evacuation and intravenous antibiotics prior to reconstruction surgery (\#1, Fig. 1 a, b), and the second one had methicillin-susceptible Staphylococcus aureus superficial infection treated with oral antibiotics (\#4, Fig. 2 a-c). Three patients had tumor-related cerebral venous thrombosis before surgery: two had partial superior sagittal sinus thrombosis (\#2 and \#4, Fig. 2 a) and had one cortical vein thrombosis (\#1, Fig. 1 A) that needed postoperative anticoagulation therapy.

All patients underwent wide scalp resection, craniectomy and dural electrocoagulation (Table 2). The mean cranial defect size was $41 \mathrm{~cm}^{2}(3.4-155)$. The 3 patients who had a dural resection required primary dural repair. Cranioplasty was performed on the same surgical stage in one patient (\#2, Fig. 3 c). Soft tissue coverage was provided by free tissue transfer of latissimus dorsi muscle in all 
140 patients (\#1, Fig. 1 c, e; \#2, Fig. 3 d; \#3, Fig. 4 e). The recipient vessels were the superficial temporal

141 artery and vein in all patients. Mean surgical duration was 7.3 (range 5 - 10.5) hours. Mean

142 intraoperative blood loss was 780 (range 400 - 1500) ml. In 4 patients (80\%) split-thickness skin graft

143 was performed in a second surgical stage few weeks later (Fig. 1 d, e; Fig. 3 d; Fig. 4 e).

\section{Postoperative management}

In the postoperative period, all patients underwent CT-scan to detect potential early

147 intracranial bleeding and then received subcutaneous heparin. Four patients had been staying for only

14824 hours in the intensive care unit, and one patient had been staying for 7 days (\#1). The median total

149 days of hospitalization was 9 (range 8 - 35) days. Four patients were discharged home and 1 was

150 discharged to a rehabilitation center before returning home.

151 Patients operated on for squamous cell carcinomas did not receive any adjuvant treatment,

152 while adjuvant radiotherapy has been performed in patients with sarcoma. A patient with systemic

153 metastases of sarcoma (\#3) received additional chemotherapy (adriamycin $60 \mathrm{mg} / \mathrm{m}^{2}$ ). Of the 5

154 patients, 1 patient who experienced an early local tumor recurrence died 7 months after surgery (\#4),

155 and 1 patient died within 10 months of surgery because of multiple systemic metastases (\#3). The

156 remaining 3 patients had no recurrence at 12, 34 and 36 months after the surgery, respectively.

157

\section{Complications}

159 There were no intraoperative complications and no complications into the donor site for the

160 tissue transfer or the skin graft. All free tissue transfers achieved 100\% soft tissue coverage.

161 Postoperative complications included one CSF leak that required lumbar punctures (\#2). Two patients

162 had flap necrosis due to arterial thrombosis (\#3 and \#5) 7 and 3 days after surgery respectively, that

163 healed after a surgical management consisting of a new free flap of latissimus dorsi. One patient

164 experienced flap dehiscence (\#4) which was treated using topical skin care. There were no

165 complications in the 4 patients who had skin grafts. 
DISCUSSION

169

The common cutaneous neoplasms of the scalp (squamous cell carcinoma, basal cell carcinoma, and malignant melanoma) are usually successfully treated with soft tissue excision, and scalp reconstruction using primary repair, local flaps or skin grafting [2, 17]. All of our patients showed invasion of their cutaneous malignancies into the cranium. This was evidenced preoperatively on imaging and confirmed intraoperatively. These patients were first recused by other local or regional hospitals because of the risk of postoperative complications and then referred to our tertiary center. Reconstruction was considerably challenging in these patients given their tumor size, and because the quality of the surrounding tissue was compromised by prior surgery, radiation or infection. Our management of these cutaneous malignancies of the scalp with deep invasion was therefore guided by the balance between obtaining clear surgical margins while avoiding neurological complications. A multidisciplinary approach has been successful in achieving complete surgical excision and immediate single-stage soft tissue reconstruction. Post-operative defect was covered by free tissue transfer of latissimus dorsi muscle with splitthickness skin graft. Other flap techniques such as rectus abdominis muscle or antero-lateral thigh were also eligible. However, free flaps are optimal for reconstruction of large skin defects [9]. In cases which require postoperative irradiation, free flap might be more reliable than local pedicle flaps.

186 Furthermore, the skin graft at the donor site may not tolerate postoperative irradiation $[6,8,16]$.

187 Latissimus dorsi muscle is a large, broad and flat muscle providing stable soft tissue coverage, 188 particularly if additional scalp needs to be resected in order to remove substrate for potential tumor recurrence [20]. The thoracodorsal artery and the thoracodorsal vein collected with this flap are of ideal caliber and length to perform the microsurgical vascular anastomosis [19]. The latissimus dorsi muscle flap also has the advantage of mimicking native scalp thickness and contour. Although secondary to the control of the malignancy, the aesthetic result of the reconstruction is an important consideration. Free flaps achieve good aesthetic results both in non-hearing and hearing areas [13]. Complications after surgery for reconstruction of scalp and calvaria in skin cancers are

195 frequent.[24] They are more frequent when skin cancer involves bone and dura mater, and 
complications include CSF leak, sinus and cortical veins thrombosis, and neurological impairment.

197 Our patients had voluminous and invasive tumors, and had poor general health condition and serious

198 comorbidities, that can explain the high rate of flap necrosis. The two cases of flap loss requiring revision surgery occurred in patients with poor general status and active smoking, the latter being a well-known risk factor for complication after free flap tissue transfer.[3, 7, 22] Special attention

201 should therefore be paid to smoking patients in the postoperative period. We recommend a systematic 202 intervention for smoking cessation before the surgery.[4] Moreover, chronic use of corticosteroids 203 does not increase rates of wound complications, reoperation, or readmission. However, this population 204 of patients may be at increased risk for major bleedings requiring blood transfusion following free flap reconstruction.[25] Superior longitudinal sinus thrombosis can occur especially in patients with tumoral involvement of the dura mater and requires pre- and/or postoperative anticoagulation therapy. Finally, patients whose surgery is delayed have a poor prognosis, suggesting that early detection and treatment are crucial.[1]

Despite flap loss in two of our patients, we obtained local healing after surgical revision. The three other patients are currently in remission. Given the severity of the initial clinical presentation, these outcomes can be considered as good. Indeed, oncological and esthetical benefits were major in patients with no other therapeutic resources. Our experienced, shared by other teams, with these advanced lesions is that even when resection is likely to be incomplete because of either sagittal sinus

214 involvement or the magnitude of the lesion, palliative resection and reconstruction are worth 215 considering $[10,14]$. We therefore advocate for an aggressive and customized surgical management 216 involving multidisciplinary collaboration and strategic thinking.

For patients with large skull defects, the indications for cranioplasty are both functional, in 218 terms of cerebral protection, and aesthetic, to maintain the contour of the calvarium. Nevertheless, performing a cranioplasty during the immediate reconstruction remains controversial, because it increases the risk of postoperative infection while it does not require a second surgery. In our series,

221 we performed immediate cranioplasty in only one patient who experienced no serious postoperative 222 adverse events. The other patients presented exophytic ulcerated tumors with concomitant 223 contamination or infection, that are contraindications for subsequent cranioplasty. In a study 
224 evaluating alloplastic cranioplasty, an infection rate of $40 \%$ was reported when calvarium was 225 reconstructed with cranioplasty in the same surgical stage [5]. Several studies reported high 226 complication rates (from 33 to 57\%), when cranioplasty was performed during the immediate 227 reconstruction, which required in most cases the removal of the prothesis [1, 3, 21]. However, these 228 studies were not exclusively on patients with cranial defects due to resection of malignant tumors. 229 Regardless of this, the higher complication rates reported in all studies about concomitant cranioplasty 230 and scalp reconstruction have to be considered with caution. Several factors appeared to be important 231 in these complications: the presence of residual devascularized or infected bone after excision, 232 inadequate coverage of the cranioplasty with well-vascularized free tissue, and the nature of the 233 material used for the cranioplasty [11, 14]. Preoperative and intraoperative recognition of the extent of 234 the bony abnormality appears therefore fundamental to guide both tumor resection and cranioplasty. 235 Cho et al. reported 11 cases of reconstruction without cranioplasty for recalcitrant invasive skin cancer 236 of the scalp and proposed to avoid cranioplasty during the immediate reconstruction [5]. Moreover, in 237 the Bethesda Cranial Facial Reconstruction Protocol delayed skull reconstruction resulted in a lower 238 complication rate than previously described [12]. This approach is based on the two major advantages 239 theoretically provided if cranioplasty is avoided at first excision. First, as seen in patients without 240 dural repair, having a vascularized graft immediately on the dura mater can limit the creation of 241 epidural fluid collections [12]. The second advantage of delaying cranioplasty is evidenced in patients 242 who underwent dural repair using xenograft material and in whom the contact with a well-vascularized 243 flap seemed to aid in healing and decreased CSF leaks [5]. Finally, although experience with 244 cranioplasty is limited, the use of titanium mesh satisfies our requirements of a malleable but sturdy 245 implant, which can be fixed rigidly. 


\section{CONCLUSION}

253

Wide resection with craniectomy, and reconstruction with free tissue transfer with or without cranioplasty provide safe and reliable treatment for invasive malignant tumors of the scalp. The free tissue transfer of latissimus dorsi muscle is the mainstay to cover complex scalp defects and tolerate radiation. The place of cranioplasty during the immediate reconstruction remains controversial and is still debated. Anyhow, the surgical management of these complex patients is a challenge that must be conducted by trained, experienced and multidisciplinary teams comprising neurosurgeons, plastic surgeons and dedicated anesthetists.

\section{Funding:}

No funding was received for this research.

\section{Conflict of Interest:}

All authors certify that they have no affiliations with or involvement in any organization or entity with any financial interest (such as honoraria; educational grants; participation in speakers' bureaus; membership, employment, consultancies, stock ownership, or other equity interest; and expert testimony or patent-licensing arrangements), or non-financial interest (such as personal or professional relationships, affiliations, knowledge or beliefs) in the subject matter or materials discussed in this manuscript.

\section{Ethical approval:}

All procedures performed in studies involving human participants were in accordance with the ethical standards of the institutional and/or national research committee (name of institute/committee) and with the 1964 Helsinki declaration and its later amendments or comparable ethical standards.

For this type of study formal consent is not required.

Informed consent was obtained from all individual participants included in the study. 
280

28

282

283

284

285

286

287

288

289

290

291

292

293

294

295

296

297

298

299

300

301

302

303

304

305

306

Patient photographic authorization:

All patients included in this study had given photographic authorization.

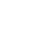




\section{REFERENCES}

308 1. Afifi A, Djohan RS, Hammert W, Papay FA, Barnett AE, Zins JE (2010) Lessons learned

309 reconstructing complex scalp defects using free flaps and a cranioplasty in one stage. J Craniofac Surg 310 21(4):1205-1209

311 2. Brandt MG, Moore CC (2019) Nonmelanoma Skin Cancer. Facial Plast Surg Clin N Am $312 \quad 27(1): 1-13$

313 3. Chao AH, Yu P, Skoracki RJ, Demonte F, Hanasono MM (2012) Microsurgical reconstruction 314 of composite scalp and calvarial defects in patients with cancer: a 10-year experience. Head Neck $31534(12): 1759-1764$

316 4. Chen D, Wu L-T (2015) Smoking cessation interventions for adults aged 50 or older: A 317 systematic review and meta-analysis. Drug Alcohol Depend 154:14-24

318 5. Cho GJ, Wang F, Garcia SM, Viner J, Hoffman WY, McDermott MW, Pomerantz JH (2017)

319 Recalcitrant Invasive Skin Cancer of the Scalp: Combined Extirpation and Microsurgical 320 Reconstruction Without Cranioplasty. J Craniofac Surg 28(2):325-330

321 6. Earley MJ, Green MF, Milling MA (1990) A critical appraisal of the use of free flaps in 322 primary reconstruction of combined scalp and calvarial cancer defects. Br J Plast Surg 43(3):283-289

323 7. Ehrl D, Heidekrueger PI, Haas EM, Coenen M, Giunta R, Ninkovic M, Broer PN (2018) Does 324 Cigarette Smoking Harm Microsurgical Free Flap Reconstruction? J Reconstr Microsurg 34(7):492325498

326 8. Furnas H, Lineaweaver WC, Alpert BS, Buncke HJ (1990) Scalp reconstruction by 327 microvascular free tissue transfer. Ann Plast Surg 24(5):431-444

328 9. Hussussian CJ, Reece GP (2002) Microsurgical scalp reconstruction in the patient with cancer.

329 Plast Reconstr Surg 109(6):1828-1834

330 10. Ioannides C, Fossion E, McGrouther AD (1999) Reconstruction for large defects of the scalp 331 and cranium. J Cranio-Maxillo-fac Surg Off Publ Eur Assoc Cranio-Maxillo-fac Surg 27(3):145-152

332 11. Kozák J, Voska P, Zverrina E (1995) The management of extensive tumours of the scalp. Acta 333 Chir Plast 37(3):71-75

334 12. Kumar AR, Tantawi D, Armonda R, Valerio I (2012) Advanced cranial reconstruction using 
335 intracranial free flaps and cranial bone grafts: an algorithmic approach developed from the modern

336 battlefield. Plast Reconstr Surg 130(5):1101-1109

337 13. Lutz BS, Wei FC, Chen HC, Lin CH, Wei CY (1998) Reconstruction of scalp defects with 338 free flaps in 30 cases. Br J Plast Surg 51(3):186-190

339 14. McCombe D, Donato R, Hofer SOP, Morrison W, Hofer S (2002) Free flaps in the treatment

340 of locally advanced malignancy of the scalp and forehead. Ann Plast Surg 48(6):600-606

341 15. McLean DH, Buncke HJ (1972) Autotransplant of omentum to a large scalp defect, with 342 microsurgical revascularization. Plast Reconstr Surg 49(3):268-274

343 16. Pennington DG, Stern HS, Lee KK (1989) Free-flap reconstruction of large defects of the 344 scalp and calvarium. Plast Reconstr Surg 83(4):655-661

345 17. Richmond HM, Duvic M, Macfarlane DF (2010) Primary and metastatic malignant tumors of 346 the scalp: an update. Am J Clin Dermatol 11(4):233-246

347 18. Rowe DE, Carroll RJ, Day CL (1992) Prognostic factors for local recurrence, metastasis, and 348 survival rates in squamous cell carcinoma of the skin, ear, and lip. Implications for treatment modality 349 selection. J Am Acad Dermatol 26(6):976-990

350 19. Serletti JM, Moran SL, Orlando GS, Fox I (1999) Thoracodorsal vessels as recipient vessels 351 for the free TRAM flap in delayed breast reconstruction. Plast Reconstr Surg 104(6):1649-1655

352 20. Sood R, Easow JM, Konopka G, Panthaki ZJ (2018) Latissimus Dorsi Flap in Breast 353 Reconstruction: Recent Innovations in the Workhorse Flap. Cancer Control J Moffitt Cancer Cent $354 \quad 25(1): 1073274817744638$

355 21. Sosin M, De la Cruz C, Bojovic B, Christy MR, Rodriguez ED (2015) Microsurgical 356 Reconstruction of Complex Scalp Defects: An Appraisal of Flap Selection and the Timing of 357 Complications. J Craniofac Surg 26(4):1186-1191

358 22. Thomas WW, Brant J, Chen J, Coblens O, Fischer JP, Newman JG, Chalian AA, Shanti RM, 359 Cannady SB (2018) Clinical Factors Associated With Reoperation and Prolonged Length of Stay in

360 Free Tissue Transfer to Oncologic Head and Neck Defects. JAMA Facial Plast Surg 20(2):154-159

361 23. Wackym PA, Feuerman T, Strasnick B, Calcaterra TC (1990) Reconstruction of massive 362 defects of the scalp, cranium, and dura after resection of scalp neoplasms. Head Neck 12(3):247-253 
363 24. Wang HT, Erdmann D, Olbrich KC, Friedman AH, Levin LS, Zenn MR (2007) Free flap 364 reconstruction of the scalp and calvaria of major neurosurgical resections in cancer patients: lessons 365 learned closing large, difficult wounds of the dura and skull. Plast Reconstr Surg 119(3):865-872

366 25. Weisberger JS, Oleck NC, Ayyala HS, Malhotra R, Lee ES (2019) Analysis of the impact of 367 chronic corticosteroid use on free flap reconstruction. Microsurgery. doi: 10.1002/micr.30516 368 


\section{FIGURE LEGENDS}

\section{Fig. 1 (patient \#1)}

394 A 35-year-old man with a history of sarcoma (diagnosed in 2000 in Algeria with 5 resection surgeries between 2000 and 2017), arrived in France in July 2017. He presented a wide skin, bone and dura mater damage (a and b) with a left extra and subdural empyema (a). A three-stage surgical management was performed. First, skin and pericranium resection with craniectomy and empyema evacuation have been performed. Postoperatively, intravenous antibiotics and anticoagulation therapy were started to threat both empyema and a cortical vein thrombosis. The second surgical stage was the reconstruction of cranial and skin defects, using a free flap of latissimus dorsi muscle (c), performed one month after the first surgical time. Finally, two weeks after the second surgical stage, a skin graft (donor-site was anterior thigh face) allowed to cover the flap (d). Lateral view of follow-up at 6 weeks 403 demonstrating stable soft tissue coverage (e).

404

\section{Fig. 2 (patient \#4)}

A 70-year-old man who developed recurrent invasive squamous cell carcinoma after immunosuppression for living-related kidney transplant. Preoperative T1-weighted magnetic resonance imaging (a) and CT scan (b) show an ulcerated tumor with invasion through the scalp into the cranium with superior longitudinal sinus tumoral involvement (c). Scalp and calvarial defect after

410 wide local excision (d). A large Surgicel sheet was used to repair the dural defect (d). The defect was 411 then reconstructed by a latissimus dorsi muscle free flap.

\section{Fig. 3 (patient \#2)}

414 A 61-year-old woman, with a long history of recurrent squamous cell carcinoma (diagnosed in 2012, 415 treated by chemotherapy and radiotherapy between 2012 and 2015, with follow-up break between 4162015 and 2017) arrived in September 2017. She presented a wide skin, bone and dura mater damage 417 with tumor invasion of the superior longitudinal sinus (a). We performed skin (b), bone (c) and dura 418 mater excision. In the same time, the defect was reconstructed with tailored titanium cranioplasty (c) 
419 and free flap of latissimus dorsi muscle. The patient had a little CSF leak after surgery resolved with

420 lumbar punctures. After three weeks, a skin graft (donor-site was antero-internal thigh face) allowed to 421 cover the flap. View of follow-up after free latissimus dorsi muscle flap with split-thickness skin graft 422 and postoperative radiation $(\mathbf{d})$.

\section{Fig. 4 (patient \#3)}

425 A 68-year-old man presented in February 2018 with a giant retro-auricular left undifferentiated 426 sarcoma (diameter was $29 \mathrm{~cm}$ ) involving skin, bone and dura mater (a and b). A preoperative 427 embolization was performed to limit bleeding during the surgery. Two days after embolization, 428 resection surgery was achieved (c and d). The defect was reconstructed in the same time with a free 429 flap of latissimus dorsi muscle. Patient had a flap necrosis, one week after surgery, requiring a revision 430 surgery with a new latissimus dorsi muscle free flap. This second surgery led to local healing (e). 